\title{
Case report of dietary supplements improving macular pigment and visual function
}

\begin{abstract}
Purpose: This case report was designed to investigate the visual, retinal, and macular pigment density (MPOD) changes in a diverse group of subjects being treated in a clinical setting with a supplement designed to increase macular pigment density, with follow-up at 6 month intervals over a 24 month time period
\end{abstract}

Methods: A pre-screening intake history was recorded to allow identification and selection of the patient population to be followed, baseline examination of the central retina, macular pigment optical density (MPOD), visual function testing, and SD-OCT imaging of the macular region were performed, and follow-up visits were performed at 6,12,18, and 24 months.

Results: Improvements were recorded in macular pigment density and visual functions, with improvement in macular appearance and changes in metamorphopsia.

Conclusion: Adding lutein/zeaxanthin/fish oil/antioxidant macular supplement with zinc, along with advice of sun protection, dietary changes and smoking recommendations improved visual functioning, and exhibited positive retinal changes documented by SDOCT and fundus photography.
Volume 6 Issue I - 2017

John P Herman, Sara J Kleiner Goudey,
Robert L Davis
Department of Optometry, Pittsfield Vision Associates, USA

Correspondence: John Herman, Department of Optometry, Pittsfield Vision Associates, 217 South Street Pittsfield, MA 0I 20I, USA, Tel 4I3-499-3797, Email eyemanage@msn.com

Received: December 01, 2016 | Published: January 19, 2017
Abbreviations: MPOD, macular pigment density; AREDS, age-related eye disease study; HPLC, high-performance liquid chromatography; HFP, heterochromic flicker photometry; LAST, lutein antioxidant supplement trial

\section{Background}

As is well known, free radicals induce cell injury. Antioxidants are the natural compensation for this process, however, when antioxidant capacity is exceeded by free radicals, oxidative stress and damage occurs. Intense light exposure has been shown to increase the rate of oxidative metabolism in the retina, resulting in high oxidative stress in eye tissues, most problematic within the retina..$^{-3}$ Exposure to light at the blue end of the visible spectrum promotes changes in and the potential for breakdown of the retinal pigment epithelium and Bruch's membrane, allowing the possible intrusion of drusen and ultimately serum and heme. This leads to the pathological processes where nonexudative age related macular degeneration progresses to exudative (wet) AMD. Animal studies have aptly demonstrated retinal damage caused by exposure to excessive blue light radiation., 4

Antioxidants in the human body consume free radicals, slowing the aging process and the deterioration in tissues, as well as aiding in the body's ability to modulate inflammation, with lutein and zeaxanthin aiding in the protection of lipid membranes, as well as in absorption of blue light, protecting the retina. ${ }^{6}$ The original Age-Related Eye Disease Study (AREDS) found a 25\% reduction in patients advancing from moderately advanced dry AMD to wet or proliferative AMD with the ingestion of an antioxidant formula with zinc. ${ }^{7}$ Epidemiological studies have shown a link between dietary and serum levels of zeaxanthin and lutein and increasing macular pigment optical density (MPOD) ${ }^{8,9}$ This has also been substantiated in donor eyes, where the levels of lutein and zeaxanthin extracted were evaluated using high- performance liquid chromatography (HPLC). Those in the highest quartile of lutein and zeaxanthin in the macular region had an $82 \%$ lower risk for AMD than those in the lowest quartile. ${ }^{10}$

Much work has been done identifying the foods highest in carotenoids, ${ }^{11,12}$ and the levels of lutein and zeaxanthin within the macular pigment layer, ${ }^{13}$ as well as the variations across the population as a function of age, sex, and ethnicity, with people of Mexican origin determined as having the highest levels of zeaxanthin, most likely due to elevated intake of foods based on corn (high zeaxanthin levels) and corn flour. ${ }^{14}$ A secondary analysis of the AREDS2 study data showed that altering the supplement used by adding zeaxanthin, lutein, and Omega 3, as well as eliminating beta-carotene and lowering the zinc level, reduced the progression to wet AMD by $35 \%$, specifically in the subgroup described as having the greatest dietary deficiency of these carotenoids. Studying a similar baseline population, and using the same fundus photo analysis technique used in the original AREDS study in order to judge efficacy of the treatment arms in reducing advancement to later stages of AMD, the lutein/zeaxanthin/ fish oil containing treatment arm showed increased ability to slow the progression of AMD over the original AREDS formula. ${ }^{15}$

Other laboratory and clinical investigations have pinpointed the specific carotenoids that constitute the protective macular pigment, those of zeaxanthin and lutein. ${ }^{16-19}$ Visual functions, including contrast sensitivity and night driving capabilities (glare recovery) have been studied by many investigators and have been shown to be correlated with elevation of MPOD scores and elevation of lutein and zeaxanthin levels in the macular pigment. ${ }^{20-29}$ Multifocal ERG has been demonstrated to quantify basic retinal cell function and can be modified by increasing levels of MPOD. The three treatment arms included $10 \mathrm{mg}$ of lutein, $20 \mathrm{mg}$ of lutein, $10 \mathrm{mg}$ lutein with $10 \mathrm{mg}$ zeaxanthin and a placebo group. ERG measurements were made in 
six concentric annular zones around the macula, and the findings were correlated with MPOD readings taken at baseline and at 6 and 12 months. The macular area associated with the central most ERG rings showed greater functional improvement with the combination zeaxanthin and lutein supplement, while the outer rings associated with the paramacular area showed best functional improvement with the $20 \mathrm{mg}$ lutein subgroup.

Of the many carotenoids that have been identified in the human body, zeaxanthin and lutein are the only two found in the eye. In the central macula, the macular pigment layer is located chiefly in the Henle fibers, whereas in the parafoveal areas are chiefly found in the inner nuclear layer. In the central macular area there is a greater density of zeaxanthin to lutein by a ratio of 2 to 1 , while in the paramacular area the ratio is reversed. ${ }^{30}$ Both zeaxanthin and lutein are necessary to maintain both the central and paracentral visual function in the human eye. Meso-zeaxanthin has also been identified in the macular area; it has been shown to be by product of lutein metabolism. ${ }^{31}$ Macular pigment densitometry has been used in many studies related to macular evaluation and AMD. It measures the macular pigment density (MPOD), utilizing a technique based upon heterochromic flicker photometry (HFP). It has been shown to accurately measure MPOD via fundus reflectance. Many reliability and repeatability studies have been cited it as the best option for measuring MPOD..$^{32,33}$

\section{Methods}

\section{Baseline risk assessment}

Participants were identified by macular degeneration risk factors to include family history, general health and medications history, central fundus findings, baseline MPOD readings, and dietary habits, with emphasis on intake of specific foods, including those known to be rich in the carotenoids, notably lutein and zeaxanthin.

\section{Patient selection}

Of the 777 patients evaluated for potential inclusion, 579 were judged to be eligible for this case report study. Within the first 30 days 58 patients discontinued. The remaining 521 patients were identified and followed for 24 months with varying degrees of these selection criteria: positive family history of macular degeneration, retinal findings known to be associated with AMD (drusen, RPE thinning, irregularities, and deposits, Bruch's membrane compromise, macular pigmentary loss/alterations), MPOD score, most below the population mean, poor diet with low intake of carotenoid rich foods, and other risk factors, such as smoking. Due to the levels of vitamin $\mathrm{K}$ in carotenoid-rich vegetables, as well as in the supplement used, patients on anticoagulant therapy were either not accepted, or were counselled relative to the dietary intake of inappropriate foods.

Approval was also obtained from their treating primary care physician in cases where it was deemed necessary or requested by the patient. If a primary care doctor was reluctant to approve participation, these patients were not enrolled. Those on anticoagulant therapy frequently introduce relatively small amounts of vitamin $\mathrm{K}$ rich vegetables (green leafy vegetables are at the top of the list, with kale, spinach, and collard greens being the top three)into their diet on a controlled basis, and thus, in these cases this treatment regimen was not significantly different than their standard diet. There were no side effects noted in this anticoagulant therapy group (9 participants). In some cases, the clotting factor was evaluated in the early stages, and in no case was a found to be an issue. . Some macular nutrition supplements have a significant level of beta-carotene, with its known lung cancer risk factor in smokers. Eye Promise Restore was the supplement used in this study, it does not contain beta-carotene. Ingredients include vitamins $\mathrm{C}$ and $\mathrm{E}, 15 \mathrm{mg}$. zinc, fish oil, alpha lipoic acid, mixed to copherols, lutein and zeaxanthin.

\section{Data collection and management}

The case report group was evaluated at baseline and again at sixmonth intervals for MPOD, best corrected visual acuity, Amsler grid and color vision testing, slit lamp examination of the posterior pole and macula. Simultaneously, posterior pole/macula fundus photos and high definition 2-D and 3-D spectral domain OCT images were recorded, allowing for side-by-side comparisons of the images, as well as counting of drusen formations. Patients included were provided dietary information with attention to lutein, zeaxanthin and omega 3intake. Nutritional supplementation with Eye Promise Restore (Zeavision) was initiated with 515 participants followed (98.8\%) and completed, while the remaining six patients reported consuming another brand of OTC supplement of their choice. Recommended dose was dependent upon intake risk evaluation scoring. As biomarkers improved, changes in dosage were recommended in some cases. As the MPOD score advanced into the $.50-.55$ range and higher, patients utilizing a full dose (two capsules per day) were sometimes reduced to a half dose, on a case-by-case basis, with consideration given to macular findings and other risk factors.

\section{Statistical analysis}

Basic descriptive statistics were calculated, with means and standard deviations being presented for those variables that were measured at baseline and monitored throughout the evaluation period. Comparisons were made using a chi-square test for categorical data, or a Fisher's exact when the assumptions for using a chi-square test were not met. When presenting information on change in MPOD from baseline, improvement was calculated as a percentage change between baseline and two years (i.e. two year MPOD /baseline MPOD). Thus, those with lowest quartile baseline MPOD scores could improve greatly on a percentage basis without as great an improvement in raw score, the converse is true for those with more elevated baseline scores. Changes in ocular and visual data were categorized as in three categories of change in MPOD: $<15 \%$ change, $15-30 \%$ change, and $>30 \%$ change, as measured at the 24 month visit.

\section{Results}

Baseline Data: (Table 1\&2): Baseline data, including age and sex of the population and hereditary and macular risk factors are shown. Overall changes in macular pigment optical density (MPOD) (Table $3 \& 4)$ : A total of 521 patients were followed for 24 months, 360 females $(69.1 \%)$ and 161 males $(30.9 \%)$, with a mean age of 53.4 years \pm 10.10 . The mean MPOD baseline score was $.2791 \pm .1260$ (range .04-.71). The mean MPOD at 24 months was .5096 .948, a mean increase of $.2305(82.6 \%)$, with $88.3 \%$ of patients showing an increase in MPOD of at least 30\%. Best corrected Snellen visual acuity changes within the 3 MPOD change categories (Table 5): Overall, patients experienced stable best corrected visual acuity (chi-square $\mathrm{p}$ value $=0.632$ ). Mean visual acuity improved from 20/30-2 at baseline to $20 / 25+2$ at 24 months. No change in best corrected visual acuity was measured in $71.4 \%$ of the participants. As has been described in many studies and is well known in both the clinical eye care and scientific communities, the incidence of factors associated with AMD is greater in females than males, and the incidence increases with age, as is seen in this study. 
Table I Study population descriptive data $n=52$ I

\begin{tabular}{ll} 
Males & I6I (30.9\%) \\
Females & $360(69.1 \%)$ \\
Age 25-30 & II (2.I\%) \\
Age 3I-40 & $30(5.8 \%)$ \\
Age 4I-50 & $139(26.7 \%)$ \\
Age 5I-60 & $222(42.6 \%)$ \\
Age 6I-70 & $89(17.1 \%)$ \\
Over 70 & $30(5.8 \%)$ \\
\hline
\end{tabular}

Table 2 Baseline risk factors Family history and retinal findings baseline data

$$
\text { One family member with AMD }
$$

$101(19.4 \%)$

Two or more family members with AMD

Hard macular drusen with amsler grid changes

Hard macular drusen without amsler grid changes

Hard paramacular drusen with amsler grid changes

Hard paramacular drusen without amsler grid changes

Soft drusen with rpe changes no amsler grid changes Soft drusen with rpe changes and amsler grid changes

Table 3 Overall change in raw MPOD data throughout the 24 month study period $n=521$

\begin{tabular}{llllll}
\hline Mean age 53.4 years & Baseline MPOD & $\mathbf{6 m}$ & $\mathbf{1 2 m}$ & $\mathbf{1 8 m}$ & $\mathbf{2 4 m}$ \\
& reading & MPOD & MPOD & MPOD & MPOD \\
& & reading & reading & reading & reading \\
\hline Mean & 0.2791 & 0.3472 & 0.4081 & 0.4653 & 0.5096 \\
Std. Deviation & \pm .1260 & \pm 1011 & \pm 988 & \pm 902 & \pm 948 \\
Range & $.04-.71$ & $.10-.72$ & $.12-.74$ & $.13-.73$ & $.10-.79$ \\
Mean \% increase & - & $24.30 \%$ & $46.20 \%$ & $66.70 \%$ & $82.60 \%$ \\
\hline
\end{tabular}

Table 4 Overall \% change in MPOD scores as a function of baseline MPOD readings throughout the 24 month study period $\mathrm{n}=52 \mathrm{I}$

\begin{tabular}{|c|c|c|c|c|}
\hline Initial MPOD reading & $\begin{array}{l}\text { 6m MPOD mean } \\
\text { increase }\end{array}$ & $\begin{array}{l}\text { I } 2 \mathrm{~m} \text { mean } \\
\text { increase }\end{array}$ & $\begin{array}{l}\text { I8m mean } \\
\text { increase }\end{array}$ & $\begin{array}{l}24 \mathrm{~m} \text { mean } \\
\text { increase }\end{array}$ \\
\hline$<.20$ & $171 \%$ & $297 \%$ & $421 \%$ & $502 \%$ \\
\hline $.21-.30$ & $91.20 \%$ & $131.40 \%$ & $166.60 \%$ & $191.20 \%$ \\
\hline $.3 \mid-.40$ & $29.20 \%$ & $56.10 \%$ & $72.60 \%$ & $84.20 \%$ \\
\hline $.4 I-.50$ & $9.40 \%$ & $13.20 \%$ & $31.10 \%$ & $49.30 \%$ \\
\hline Over .50 & $6.70 \%$ & $11.90 \%$ & $21.20 \%$ & $20.40 \%$ \\
\hline Overall Range & $6.7 \%-171 \%$ & II.9\% - $-297 \%$ & $12.2 \%-421 \%$ & $13.2 \%-502 \%$ \\
\hline Mean \% Increase All Categories & $24.30 \%$ & $46.20 \%$ & $66.70 \%$ & $82.60 \%$ \\
\hline
\end{tabular}

Table 5 Change in best corrected visual acuity (BCVA) as a function of degree of MPOD change $n=52$ I

\begin{tabular}{|c|c|c|c|c|}
\hline \multirow[t]{3}{*}{ BCVA change } & \multicolumn{4}{|c|}{ MPOD improvement categories } \\
\hline & $<15 \%$ & $15 \%$ to $30 \%$ & $>30 \%$ & Total \\
\hline & $n(\%)$ & $n(\%)$ & $n(\%)$ & \\
\hline \multirow[t]{2}{*}{ No change } & 23 & 18 & 331 & 372 \\
\hline & $-82.10 \%$ & $-54.60 \%$ & $-72.00 \%$ & $-71.40 \%$ \\
\hline Improvement & 4 & 12 & 96 & 112 \\
\hline (3-5 letters) & $-14.30 \%$ & $-36.40 \%$ & $-20.90 \%$ & $-21.50 \%$ \\
\hline Significant Improvement & I & 3 & 33 & 37 \\
\hline (6- 12 letters) & $-3.60 \%$ & $-9.10 \%$ & $-7.20 \%$ & $-7.10 \%$ \\
\hline \multirow[t]{2}{*}{ Totals } & 28 & 33 & 460 & 521 \\
\hline & $0.05 \%$ & $0.06 \%$ & $88.30 \%$ & $-100 \%$ \\
\hline
\end{tabular}


It is also known that family history is a risk factor for AMD, as is the appearance of drusen in the posterior pole. In this study, 33\% reported a family history of one or more forms of AMD. Hard drusen were seen in the macular and/or paramacular areas in $70.2 \%$ of participants, the number and density of drusen varied from 1-2 to as many as $50-60$, with $19.1 \%$ with positive Amsler Grid findings. The hard drusen were easily counted and followed with serial SD-OCT evaluations, allowing for side-by-side comparison of consecutive images, making changes in OCT images relatively easy to determine. Baseline soft drusen with macular depigmentation and RPE changes were seen in $22.8 \%$ of the population $(82.4 \%$ with Amsler Grid changes being recorded). Following this group with SD-OCT was not quite as easy, as the varying density of the soft-edged drusen along with their tendency to coalesce and vary in color make them more difficult to define and count as compared to the more well-defined hard drusen, as one might expect.
In $21.5 \%$, an improvement of $3-5$ letters was measured, and in $7.1 \%$ an improvement of 6-12 letters was recorded at the final 24 month visit, a greater improvement in visual acuity was shown in other studies previously cited. Changes in visual acuity did not show any significant correlation with MPOD improvement category, as the number of participants with small MPOD improvement was not significant enough to draw any conclusions. Changes in visual functions: (Table 6\&7): Participants reported subjective changes in visual function at the one and two year follow-up visits. Visual function changes were reported specifically in two categories, perceived change in glare reduction/recovery with night driving, and improvement in visual capabilities in low light levels/low contrast situations, factors affecting the daily lives of people, more often reported and noteworthy with aging.

Table 6 Reported change in glare recovery at the 24 month follow-up visit as a function of degree of MPOD improvement, (Improved night driving capabilities) $\mathrm{n}=521$

\begin{tabular}{|c|c|c|c|c|}
\hline \multirow[t]{2}{*}{ Reported change in glare recovery with night driving } & \multicolumn{4}{|c|}{ MPOD improvement categories } \\
\hline & $\begin{array}{l}<15 \% \\
\text { MPOD } \\
\text { improvement }\end{array}$ & $\begin{array}{l}15-30 \% \text { MPOD } \\
\text { improvement }\end{array}$ & $\begin{array}{l}>30 \% \\
\text { MPOD } \\
\text { improvement }\end{array}$ & $\begin{array}{l}\text { Total } \\
\text { n(\%) }\end{array}$ \\
\hline No change & $\begin{array}{l}5 \\
(17.9 \%)\end{array}$ & $\begin{array}{l}15 \\
(45.5 \%)\end{array}$ & $\begin{array}{l}147 \\
(32.0 \%)\end{array}$ & $\begin{array}{l}167 \\
(32.1 \%)\end{array}$ \\
\hline Improvement & $\begin{array}{l}7 \\
(25.0 \%)\end{array}$ & $\begin{array}{l}3 \\
(9.1 \%)\end{array}$ & $\begin{array}{l}183 \\
(39.8 \%)\end{array}$ & $\begin{array}{l}193 \\
(37.0 \%)\end{array}$ \\
\hline Significant improvement & $\begin{array}{r}16 \\
(57.1 \%)\end{array}$ & $\begin{array}{l}15 \\
(45.5 \%)\end{array}$ & $\begin{array}{l}130 \\
(28.3 \%)\end{array}$ & $\begin{array}{l}161 \\
(30.9 \%)\end{array}$ \\
\hline $\begin{array}{l}\text { Totals in improvement } \\
\text { categories }\end{array}$ & $\begin{array}{l}23 \\
(82.1 \%)\end{array}$ & $\begin{array}{l}18 \\
(75.5 \%)\end{array}$ & $\begin{array}{l}313 \\
(68 \%)\end{array}$ & $\begin{array}{l}354 \\
(67.9 \%)\end{array}$ \\
\hline
\end{tabular}

Table 7 Reported improvement in visual function in low contrast visual environment situations as a function of degree of MPOD change $n=521$

\begin{tabular}{|c|c|c|c|c|}
\hline \multirow[t]{2}{*}{ Improvement in visual function in low contrast visual environment } & \multicolumn{4}{|c|}{ MPOD change categories } \\
\hline & $\begin{array}{l}<15 \% \\
\text { n (\%) }\end{array}$ & $\begin{array}{l}15 \%-30 \% \\
\text { n (\%) }\end{array}$ & $\begin{array}{l}>30 \% \\
\text { n (\%) }\end{array}$ & $\begin{array}{l}\text { Total } \\
\mathrm{n}(\%)\end{array}$ \\
\hline No reported change & $\begin{array}{l}12 \\
(42.9 \%)\end{array}$ & $\begin{array}{l}24 \\
(72.7 \%)\end{array}$ & $\begin{array}{l}162 \\
(35.2 \%)\end{array}$ & $\begin{array}{l}198 \\
(38.0 \%)\end{array}$ \\
\hline Improved & $\begin{array}{l}7 \\
(25.0 \%)\end{array}$ & $\begin{array}{l}\text { I } \\
(3.0 \%)\end{array}$ & $\begin{array}{l}81 \\
(17.6 \%)\end{array}$ & $\begin{array}{l}89 \\
(17.1 \%)\end{array}$ \\
\hline Significantly improved & $\begin{array}{l}9 \\
(32.1 \%)\end{array}$ & $\begin{array}{l}8 \\
(24.2 \%)\end{array}$ & $\begin{array}{l}217 \\
(47.2 \%)\end{array}$ & $\begin{array}{l}234 \\
(44.9 \%)\end{array}$ \\
\hline $\begin{array}{l}\text { Total improved } \\
\text { (\%) }\end{array}$ & $\begin{array}{l}16 \\
\text { improved } \\
(3.1 \%)\end{array}$ & $\begin{array}{l}9 \\
\text { improved. } \\
(.02 \%)\end{array}$ & $\begin{array}{l}298 \\
\text { improved } \\
(57.2 \%)\end{array}$ & $\begin{array}{l}323 \\
\text { improved } \\
(62 \%)\end{array}$ \\
\hline
\end{tabular}

360 females (69.1\%) 161 males $(30.9 \%)$. A steady improvement in macular pigment density (MPOD) was shown over the 24 month study period, with the largest improvement percentage seen in those with very poor baseline readings. All patients showed improvement in MPOD readings over the 24 month study. Overall changes in macular pigment optical density (MPOD) (Tables 3\&4): A total of 521 patients were followed for 24 months, 360 females $(69.1 \%)$ and 161 males (30.9\%), with a mean age of 53.4 years \pm 10.10 . The mean MPOD baseline score was $.2791 \pm .1260$ (range .04-.71). The mean MPOD at 24 months was $.5096 \pm .948$, a mean increase of $.2305(82.6 \%)$, with $88.3 \%$ of patients showing an increase in MPOD of at least $30 \%$.

360 females (69.1\%) 161 males (30.9\%), Mean age 53.4 years. A large range in percentage of improvement in macular pigment density (MPOD) over the 24 month study period, inversely proportional to the baseline flicker photometry MPOD measurements. There 
were no patients who did not show at least minor improvement in MPOD readings. Stability in best corrected visual acuity, as measured on a standard clinical Snellen chart, was most common, there was improvement measured in all three categories of MPOD improvement, ranging between 3 letters on a standard Snellen chart and $2+$ lines (12 letters). All were measured prior to pupillary dilation with best correction in place. Obviously, other factors can affect any subjective test results, including visual acuity, and need not be enumerated, but the relationship to MPOD improvement, retinal improvement, and acuity improvement, albeit to a small degree, exists.

Best corrected Snellen visual acuity changes within the 3 MPOD change categories (Table 5): Overall, patients experienced stable best corrected visual acuity (chi-square $\mathrm{p}$-value $=0.632$ ). Mean visual acuity improved from 20/30-2 at baseline to 20/25+2 at 24 months. No change in best corrected visual acuity was measured in $71.4 \%$ of the population $(\mathrm{n}=372)$. In $21.5 \%(\mathrm{n}=112)$ of the population, an improvement of 3-5 letters was measured, and in $7.1 \%(n=37)$ an improvement of 6-12 letters was recorded at the final 24 month visit, a greater improvement in visual acuity was shown in other studies.13, 14 Changes in visual acuity did not show any significant correlation with MPOD improvement category, as the number of participants with small MPOD improvement was not significant enough to draw any conclusions.

These have been studied, presented, and discussed as being a function of macular pigment density in many publications, using terms such as discomfort glare or photophobia, disability glare, which is decreased with elevated intake of lutein and zeaxanthin, photo stress recovery (the time needed to recover visual function after intense light exposure), and contrast enhancement, or improved contrast sensitivity, all of which contribute to visual viability. The data summary collected at the two year final visit is shown in these tables, presented as a function of the 3 categories of degree of MPOD improvement. The participants rated these changes in visual function in 4 categories; worse, unchanged, improved, and significantly improved. No participants reported worse visual function capabilities, thus it is not seen as a category in Tables $6 \& 7$.

An improvement in glare recovery was reported by $67.9 \%$ of the study population, most notably associated with night driving improvement, a major complaint in the population in general, particularly common in the aging population. Table 6 Perceived change in glare reduction/recovery with night driving was reported as improved by $37.0 \%$ of participants, while $30.9 \%$ reported significant improvement, for a total of $67.9 \%$ of participants reporting levels of improvement in glare reduction/recovery. MPOD improvement with reported glare reduction was not statistically significant (chi-square $\mathrm{P}$-value $<0.001$ ) as MPOD improvement from baseline was less than $30 \%$ in $11.7 \%$ of the population.

A common complaint in the overall patient population, usually increasing with age, is visual difficulty in reduced illumination situations, related to contrast sensitivity reduction, as well as other visual/ocular contributing factors. As part of the interview at each follow-up visit, patients were asked to determine and rate any changes noted. There was improvement in visual functioning reported by patients throughout the study. As this data shows, patients with a greater degree of improvement in macular pigment density (MPOD) reported greater improvements in visual functioning in low contrast visual environments. This improvement in contrast sensitivity has been reported in other studies.
Table 7 The data describing reported visual capabilities in low contrast visual environments is shown. Once again, improvement was reported by the majority $(62 \%)$, and the total number of participants in whom MPOD improvement was not greater than 30\% from baseline was very small (11.7\%), analyzing the relationship of MPOD improvement with reported improvement in visual function in low contrast situations was not statistically meaningful (chi-square $\mathrm{P}-$ value $<0.001)$.

Table 6 Perceived change in glare reduction/recovery with night driving was reported as improved by $37.0 \%$ of participants, while $30.9 \%$ reported significant improvement, for a total of $67.9 \%$ of participants reporting levels of improvement in glare reduction/ recovery. MPOD score correlation with reported glare reduction was not statistically significant (chi-square P-value $<0.001$ ), as MPOD improvement was less than $30 \%$ from base line in $11.7 \%$ of the population. Table 7 The data describing reported visual capabilities in low light and/or low contrast visual environments is shown. Improvement was reported by the majority (62\%), and the total number of participants in whom MPOD improvement was not greater than $30 \%$ from baseline was very small $(11.7 \%)$, thus analyzing the relationship of MPOD improvement with reported improvement in visual function in low contrast situations was not statistically meaningful (chi-square $\mathrm{P}-$ value $<0.001$ ), but was subjectively more than meaningful to the patients, as it was continually reported at each follow-up visit.

Table 8 Hard drusen were found in 196 patients at baseline, located in the macular and/or paramacular zones. Changes in drusen were defined by changes in the number of drusen, the size of the drusen, and/or a change in the RPE associated with these changes. Throughout the 24 month evaluation, changes in drusen were rated via side-byside comparative evaluations of SD-OCT images. Improvement was recorded in 53 patients $(27.04 \%)$, while stability was seen in $140(71 \%)$. Deterioration was seen in 2 patients (1\%). Those with an improvement in MPOD greater than 30\% experienced the greatest reduction in hard drusen count. Change in objective ocular findings (hard and soft drusen) were not necessarily related to the degree of improvement in MPOD (Tables 5\&6), all comparisons Fisher's exact, $\mathrm{P}$-value $>0.05$ ).

Central and paracentral SD-OCT evaluations throughout the study showed that, of the 199 patients in which hard drusen were seen at baseline evaluations, $53(27.0 \%)$ showed a decrease in number and/or density of drusen, and $140(71.4 \%)$ showed no discernible change, for a total of $193(98.5 \%)$ showing stability or reduction in paramacular hard drusen. No decrease in drusen count were found until the 12 month follow-up visit, increasing further after that time.

Table 8 Hard drusen were found in 196 patients at baseline, located in the macular and/or paramacular areas. Changes in drusen were defined by the number of drusen, the size of the drusen, or a change in the RPE. Through the 24 month evaluation changes in drusen were observed with SD-OCT imaging resulting in $27.04 \%(\mathrm{n}=53)$ improved, $71 \%(\mathrm{n}=140)$ remained stable, and $1 \%(\mathrm{n}=2)$ showed a progression and $.005 \%(\mathrm{n}=1)$ showed a notable expansion. In figures $2-4$, changes can be seen in the RPE typical of those seen in a small percentage of subjects studied; the majority was changes in small hard drusen, best seen in the macula as opposed to the paramacular areas, perhaps due to increased contrast with the darker macular pigment. However, the most significant changes in the macula, particularly in the RPE, as depicted with SD OCT imaging, were seen in those with 
more advanced stages with soft drusen. Those with an improvement in MPOD greater than 30\% experienced the greatest decrease in the number of hard drusen. Change in objective ocular findings (hard and soft drusen) were not necessarily related to the degree of improvement in MPOD (Tables 8\&9), (all comparisons Fisher's exact, P-value > $0.05)$.

Table 8 Changes in macular and paramacular hard drusen OCT measurements/observations as a function of degree of MPOD change $\mathrm{n}=196$

\begin{tabular}{lllll}
\hline Hard drusen change++ & MPOD change categories & & \\
Observed change in hard drusen & $<15 \%$ & $\begin{array}{l}15 \%-30 \% \text { change } \\
\mathbf{n}(\%)\end{array}$ & $\begin{array}{l}>30 \% \text { change } \\
\mathbf{n}(\%)\end{array}$ & $\begin{array}{l}\text { Totals } \\
\mathbf{n}(\%)\end{array}$ \\
\hline Improvement & $3 \%)$ & 6 & 44 & 53 \\
Stable & $(18.8 \%)$ & $(35.3 \%)$ & $(27.0 \%)$ & $(27.0 \%)$ \\
& 13 & 11 & 116 & 140 \\
Minor advancement & $(81.3 \%)$ & $(64.7 \%)$ & $(71.2 \%)$ & $(71.4 \%)$ \\
& 0 & 0 & 2 & 2 \\
Notable advancement & $(0.0)$ & $(0.0)$ & $(1.2 \%)$ & $(1.0 \%)$ \\
& 0 & 0 & 1 & 1 \\
Total stable or improved & $(0.0)$ & $(0.0)$ & $(0.6 \%)$ & $(0.5 \%)$ \\
\hline
\end{tabular}

++ No hard drusen seen in 325 participants (62.4\%)

Table 9 Changes in soft macular and/or paramacular drusen as a function of degree of MPOD change $n=119$

\begin{tabular}{|c|c|c|c|c|}
\hline \multirow[t]{2}{*}{ Soft drusen change ++ } & \multicolumn{4}{|c|}{ MPOD change categories } \\
\hline & $\begin{array}{l}<=15 \% \\
\text { n (\%) }\end{array}$ & $\begin{array}{l}>15 \% \text { to } 30 \% \\
\text { n (\%) }\end{array}$ & $\begin{array}{l}>30 \% \\
\text { n (\%) }\end{array}$ & $\begin{array}{l}\text { Total } \\
\text { n (\%) }\end{array}$ \\
\hline Improved & $\begin{array}{l}\text { I } \\
(14.30 \%)\end{array}$ & $\begin{array}{l}\text { I } \\
(9.10 \%)\end{array}$ & $\begin{array}{l}14 \\
(14.40 \%)\end{array}$ & $\begin{array}{l}16 \\
(13.90 \%)\end{array}$ \\
\hline Stable & $\begin{array}{l}6 \\
(85.70 \%)\end{array}$ & $\begin{array}{l}10 \\
(90.90 \%)\end{array}$ & $\begin{array}{l}82 \\
(84.50 \%)\end{array}$ & $\begin{array}{l}99 \\
(85.20 \%)\end{array}$ \\
\hline Advancement & $\begin{array}{l}2 \\
(<2 \%)\end{array}$ & $\begin{array}{l}1 \\
(<1 \%)\end{array}$ & $\begin{array}{l}1 \\
(<1 \%)\end{array}$ & $\begin{array}{l}4 \\
(3.40 \%)\end{array}$ \\
\hline Total stable or improved & $\begin{array}{l}7 \\
(5.90 \%)\end{array}$ & $\begin{array}{l}\text { II } \\
(9.20 \%)\end{array}$ & $\begin{array}{l}96 \\
(80.70 \%)\end{array}$ & $\begin{array}{l}115 \\
(96.60 \%)\end{array}$ \\
\hline
\end{tabular}

++ No Soft Drusen Seen in 402 Patients (77.2\%)

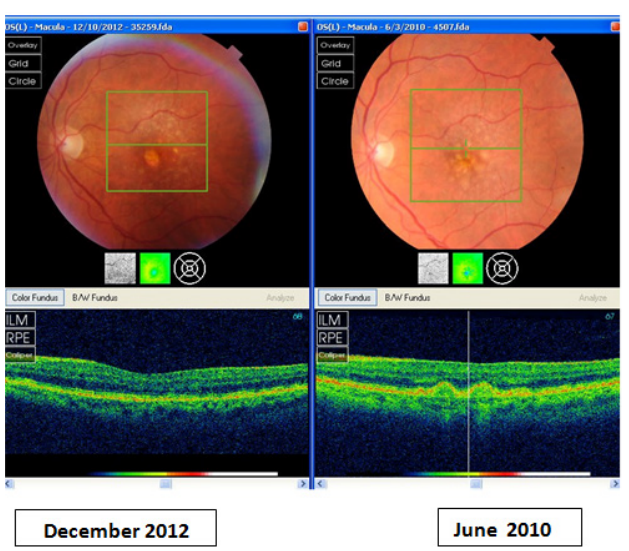

Figure I Representative of RPE changes seen in patient with hard and soft macular and paramacular drusen. Note decrease in number and shrinkage of drusen and associated improvement in RPE.

Citation: Herman JP, Goudey SJK, Davis RL. Case report of dietary supplements improving macular pigment and visual function. Adv Ophthalmol Vis Syst. 2017;6(I):24-35. DOI: 10.15406/aovs.2017.06.00166 


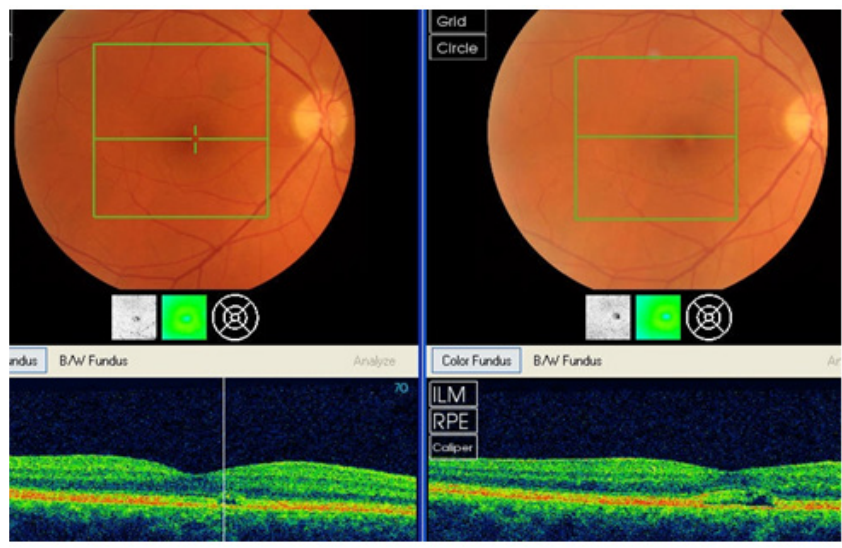

March 2012

January 2010

Figure 2 Changes in RPE of a single large drusen formation over at 2.2 year period.
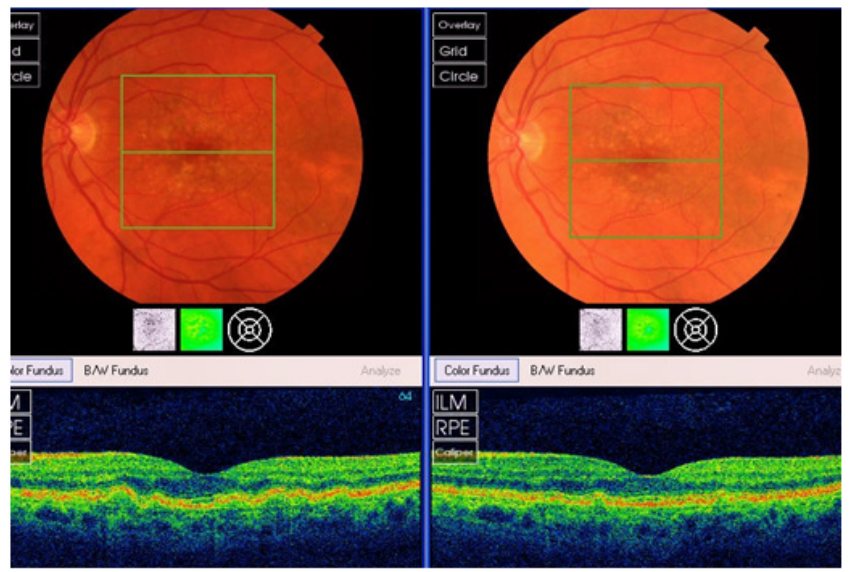

Colar Funden BN Funden

December 2009

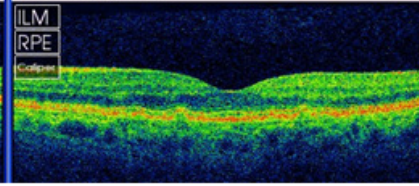

Figure 3 Overall improvement in the size, density, and number of drusen is seen over a 26 month time period. Most improvement chiefly noted within the central macular zone, with associated RPE improvement.

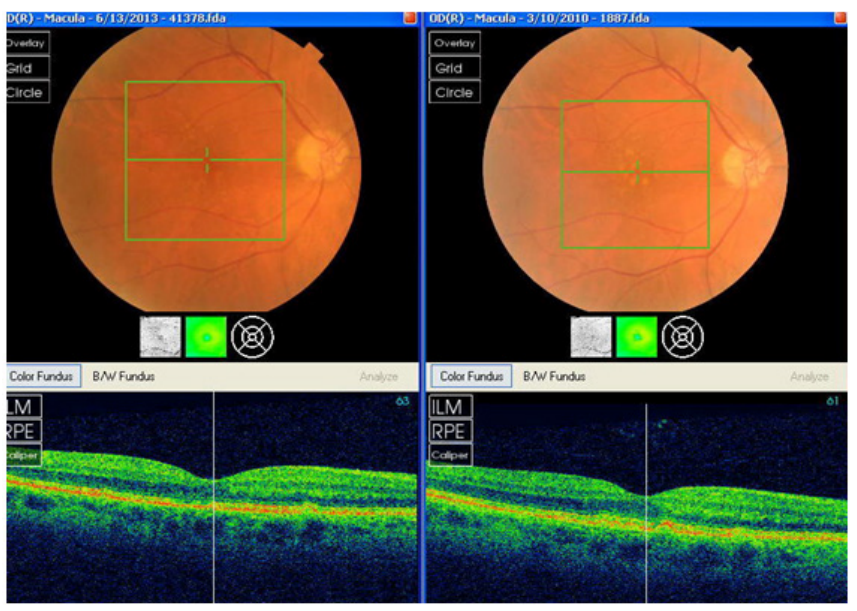

June 2013

March 2010

Figure 4 Typical of the macular changes seen in those with soft drusen concentrated in the central macular zone. Replenishment of the RPE with reduction and clearing of the central drusen over a period of 3.25 years. 
A very important factor to consider when assessing the percentage improvement in MPOD is to consider quartile category of MPOD at baseline, and its effect on the degree of change throughout the 24 month evaluation period, which can also affect all of the data based on this baseline data. In those with the most elevated baseline MPOD readings, a lower percentage of MPOD improvement is shown in follow-up evaluations. There is a difference in the percentage of patients that improved significantly (greater than 30\%) based upon the quartile of MPOD at baseline (chi-square $<0.001$ ). Overall, improvement of over $30 \%$ in MPOD was measured in $88 \%$ of the population, and greater than $15 \%$ in $95 \%$ of the population. The percentage of patients in the highest MPOD quartile at baseline represented the vast majority of patients who did not improve by at least $30 \%$ over the two years of the study.
This might be expected, and, although not totally proven, might lead one to conclude that there is perhaps an upper limit to the level of MPOD improvement that one might expect in the general population as a result of improvement in dietary habits and/or supplementation over a two year period of time. Due to this factor, percentage improvement as compared to baseline may not be an adequate descriptor of the degree of change, as those with higher baseline MPOD readings could quite possibly have healthier macular tissues, with less room for improvement (Tables 9\&10). Significant data is shown for two subgroups. One included patients who were part of this study, and another that refused to participate or dropped out of the study very early, within thirty days. Table 11 This group of participants included in study that reported at the outset that they had a very poor dietary intake of vegetables on a daily basis.

Table 10 Amsler Grid changes for those with observable metamorphopsia at baseline ++

\begin{tabular}{|c|c|c|c|}
\hline Baseline finding & $\mathbf{n}=$ & I2m Change++ & $24 m$ Change++ \\
\hline Hard macular drusen with amsler grid distortion & $\begin{array}{l}27 \\
(5.2 \%)\end{array}$ & $\begin{array}{l}\text { Improved } 2(7.4 \%) \\
\text { Stable } 24(88.8 \%) \\
\text { Advanced I (4.0\%) }\end{array}$ & $\begin{array}{l}\text { Improved I } 6 \text { (59.3\%) } \\
\text { Stable II (40.7\%) } \\
\text { Advanced } 0\end{array}$ \\
\hline Hard paramacular drusen with amsler grid distortion & $\begin{array}{l}43 \\
(8.3 \%)\end{array}$ & $\begin{array}{l}\text { Improved II (25.6\%) } \\
\text { Stable 3I }(72.1 \%) \\
\text { Advanced I }(2.3 \%)\end{array}$ & $\begin{array}{l}\text { Improved I } 7 \text { (39.5\%) } \\
\text { Stable } 26(60.5 \%) \\
\text { Advanced } 0\end{array}$ \\
\hline Soft drusen with rpe changes and amsler grid distortion & $\begin{array}{l}98 \\
(18.8 \%)\end{array}$ & $\begin{array}{l}\text { Improved } 21 \text { (2I.4\%) } \\
\text { Stable } 74(75.5 \%) \\
\text { Advanced } 3(3.1 \%)\end{array}$ & $\begin{array}{l}\text { Improved } 39(39.8 \%) \\
\text { Stable } 52(53.1 \%) \\
\text { Advanced } 7(7.1 \%)\end{array}$ \\
\hline
\end{tabular}

Table I I Change in MPOD readings over the 24 Month in the subgroup reporting habitual low degree of vegetable intake on a daily basis at baseline visit $\mathrm{n}=95$

\begin{tabular}{lllllll}
\hline Baseline MPOD reading & 6m & $12 \mathrm{~m}$ & $18 \mathrm{~m}$ & $24 \mathrm{~m}$ & \\
& Patient & Mean \% & Mean \% & Mean \% & Mean \% & Range of \\
& count & MPOD & MPOD & MPOD & MPOD & $\%$ MPOD \\
& & increase & increase & increase & increase & increase \\
\hline$<.10$ & 16 & $44.20 \%$ & $175 \%$ & $244.20 \%$ & $424.60 \%$ & $236-524 \%$ \\
$.11-.15$ & 47 & $32.30 \%$ & $174.10 \%$ & $244.30 \%$ & $361.50 \%$ & $120-411 \%$ \\
\hline
\end{tabular}

Of the 119 of patients in whom soft drusen were observed at baseline ( $22.8 \%$ of the total population), the condition, as determined via serial comparisons of SD OCT imaging, was determined to be stable in $85.2 \%$ of this population, while improvement was seen in $13.9 \%$, chiefly in the group with the greatest improvement in macular pigment density. Once again, these improvements were seen and best identified later in the study, at the 18 and 24 month visits.

Percentage change is relative to those with drusen at baseline, column 2 Amsler Grid changes were seen to improve by the 24 month visit in $42.9 \%$ of those for whom metamorphopsia was detected at the baseline visit, none of which were totally eliminated, while $53.9 \%$ showed stability with no advancement, and advancement in metamorphopsia, seen totally in the group in whom soft drusen and RPE changes were at baseline was observed (4.2\%). None of the population with hard drusen at baseline advanced in metamorphopsia as detected with the Amsler Grid, and no newly-developed Amsler Grid metamorphopsia was recorded during the study.

A subgroup evaluated were those who reported minimal vegetable intake on a daily basis at baseline. These participants were treated with the supplement only, as they did not improve their diet, and all but one of the 21 that were smokers refused to stop doing so. The results appear above. All subjects were asked to review, at each 6 month follow-up visit, their dietary habits data collected at baseline, and report any significant changes. This group reported no significant increase in their vegetable intake in their daily diet, with particular attention to vegetables with elevated carotenoid levels. The baseline MPOD measurements in this group were all below that of the overall study population mean, and lower than is recommended as the minimum safe zone for macular health by the product manufacturer. The data is shown for three categories of initial MPOD readings. All three showed significant increase in macular pigment density throughout the study despite no dietary improvements, testimony to the efficacy of the macular pigment supplement used by these patients. As demonstrated in other tables, those with the lowest baseline MPOD readings proved to benefit the most, with the highest percentage improvement in macular pigment density, as well as improvements in retinal findings and visual function measures.

Nearly all in this group were males, and this history was reported or confirmed by their spouses in most cases. The use of the supplement alone in this group elevated their MPOD readings significantly, suggesting that it is a very useful and powerful supplement that can 
and does build the density of the protective macular pigment. Table 12 describes a group that did not participate in the study. This group indicated that they thought they could manage their low macular pigment density by simply increasing the dietary intake of the recommended carotenoid-rich vegetables. This was not the case, as both groups changes minimally, with the males decreasing slightly in
MPOD ( $-3.4 \%$ at 24 months), and the females increasing $(+4.2 \%)$. While this was not, by design, a control group, it served quite well as one, as they did not consume a macular pigment supplement. The results certainly could bring into question their reporting of change in dietary habits.

Table 12 Percent change in MPOD over a 24 month period in patients refusing participation in study protocol and reporting self-management with dietary increase in carotenoid-rich vegetable intake on a daily basis $\mathrm{n}=\mathrm{I}$ I I

\begin{tabular}{lllll}
\hline Sex & $\begin{array}{l}6 \mathrm{~m} \\
\text { MPOD } \\
\text { change }\end{array}$ & $\begin{array}{l}12 \mathrm{~m} \\
\text { MPOD } \\
\text { change }\end{array}$ & $\begin{array}{l}18 \mathrm{~m} \\
\text { MPOD } \\
\text { change }\end{array}$ & $\begin{array}{l}\mathbf{2 4 m} \\
\text { MPOD } \\
\text { change }\end{array}$ \\
\hline $\begin{array}{l}\text { Males } \\
(69)\end{array}$ & $+2.1 \%$ & $+3.1 \%$ & $-1.6 \%$ & $-3.4 \%$ \\
$\begin{array}{l}\text { Females } \\
(42)\end{array}$ & $+3.6 \%$ & $+4.1 \%$ & $+3.9 \%$ & $+4.2 \%$ \\
\hline
\end{tabular}

Of the 111 patients choosing not to use the recommended macular supplement but reporting for 6 month follow-up visits, indicating that they would improve their macular pigment density by increasing their daily intake of carotenoid-rich vegetables, did not fare well, particularly the males in the group. In all probability, this group was overzealous in reporting their improvement in vegetable intake, as the MPOD readings throughout the study did not correlate with the reports of improved diet. The overall change in this subgroup was minimal, whereas the results in the 521 subjects studied showed an MPOD increase of $82.6 \%$. 20+ times greater than this group.

In Figures 1-4, stark changes can be seen in the RPE, typical of those seen in a relatively small percentage of participants, most changes in RPE were slight to moderate, and were chiefly associated with changes in small hard drusen, best seen in the macula as opposed to the paramacular areas, perhaps due to increased contrast with the darker macular pigment. However, the most significant changes in the macula, particularly in the RPE, as depicted with SD-OCT imaging, were seen in those with more advanced stages of soft drusen, as demonstrated in Figures 1-4.

\section{Discussion}

This case report lends support to the previously cited studies that have demonstrated that supplements containing lutein, zeaxanthin, antioxidants, EPA/DHA with zinc increase MPOD, improve overall contrast sensitivity, night driving, and vision in low contrast situations, while reducing glare sensitivity. The data shown in Table 11 is noteworthy, as it demonstrates the ability of the nutritional supplement to elevate the macular pigment density in a population with low intake of carotenoid-rich foods; this is responsible for the data showing the highest percentage increase in MPOD being shown in the group with the lowest baseline MPOD readings.

The data in Table 12 represents the inability of reported improvement in dietary increase of carotenoid-rich vegetables, as a sole nutritional change factor, to affect improved MPOD levels in this subgroup, at least not on a short-term basis, perhaps a longer study may prove otherwise. That does not mean that dietary and general health recommendations and counselling should not be part of the care for patients at high risk for macular degeneration, as shown in many previously cited studies, as the results of the baseline nutrition survey, in general, correlated positively to the degree of MPOD levels at baseline, although retinal compromise, general health, or family history factors warranted their inclusion in the study. Those with the highest dietary intake of foods containing higher levels of lutein, zeaxanthin and omega 3 had the highest baseline MPOD scores.

Patients that started with the lowest MPOD levels and poor initial diet intake did have the greatest success in elevating the MPOD levels with the use of the supplement, also found in many other previously cited studies. These results not only are testament to the efficacy of the supplement, but also may suggest the need of a larger long term prospective interventional study to determine if improvements in MPOD would lower the potential risk of AMD. The retinal observations and improvements in visual functions in this case report would suggest that, as well as the results of the AREDS 2 study, the LAST studies, the CAREDS study (all previously cited), and many others.

Two welcomed significant findings in this study were somewhat unanticipated at the outset, probably due to the fact that data covering the topics had not been widely published at that time. First, $62-68 \%$ of patients showed an improvement in visual function related to glare recovery and contrast sensitivity (Tables $6 \& 7$ ). This is very similar to the data reported in the previously cited Lutein Antioxidant Supplement Trial (LAST) study, which studied a somewhat different population, but with similar results. In that study, patient with macular degeneration received lutein or lutein plus additional antioxidants and demonstrated significant improved MPOD, glare recovery, near visual acuity, and contrast sensitivity function. The lutein and additional antioxidants group demonstrated a broader effect although the lutein alone group did show improvement in Amsler grid scotomas, metamorphopsia, and visual acuity.

The outcome was very similar to this evaluation within a typical clinical population seeking primary eye care that was studied in the group followed in this evaluation, in that the greatest degree improvement was found in the lowest quartile MPOD at baseline. The second unanticipated finding was the improvement in hard and soft drusen in the posterior pole associated with improving MPOD scores throughout the study. Eyes with notable hard discrete drusen showed the greatest improvements with OCT evaluation (Table 8). Changes in soft drusen, particularly coalesced soft drusen, are more difficult to evaluate, as they are less discrete (Table 9). If one accepts the concept that these initial hard drusen in the macular and paramacular areas 
are an early sign of onset of dry AMD, it could be suggested that this type of nutritional intervention very early in the disease process could result in better ability to positively affect the disease process, and could be preventive.

An associated retinal finding that requires further investigation is the notable reduction in the irregularities in the retinal pigment epithelium that can be seen in some cases, associated with these changes in drusen, and at times it was the most noteworthy and significant change seen (see the attached Figures 1-4). It is difficult to objectively score these observations, but side-by-side evaluations with the (Topcon 2000) SD-OCT, shows it to be unmistakably present. There is a great deal of difference in the appearance of the RPE in the subgroups of patients, in general, thicker and uniform RPE is seen in patients with elevated MPOD scores, although media and other changes can inhibit totally accurate evaluation, and while this was evaluated, the data is not included as part of this evaluation, chiefly due to the wide descriptive categories encountered.

Subjective reports of visual function changes were separated into three categories, little or no change, improvement, and significant improvement. These categories are dependent upon subjective reporting, and while they are very important clinically and to the visual functioning of an individual patient, subjective reporting does they do not have a purely scientific basis and can thus be somewhat difficult to analyze statistically, although these results match up very closely with data from many other previously cited studies. Such is the case with all subjective reports, whether the patient is reporting changes in visual function, sensation with dry eyes, pain levels, etc. Thus, one must consider that, while patients who showed improvements in MPOD readings in general showed some form of improvement in visual capabilities, the lines between the patients categorization of these changes are subjective, and can thus not be strictly analyzed, like those with numeric quantities attached to them, such as MPOD readings. Nevertheless, this data very strongly suggests that this relationship exists, has done so in studies previously cited, and most patients who experience these improvements in visual function indicated that these changes were very significant to them.

One of the shortcomings of the initial AREDS study was the lack of SD-OCT technology that could have permitted more precise categorization of biomarkers through serial OCT scans of the macular region. Lack of OCT data required the biomarker of maintenance of the dry form of AMD to be the significant data point, with analysis at macular photo reading centers. The data supplied in the AREDS studies was very significant, as it looked at the more advanced cases of dry and wet AMD, and obviously spurred much more research in this field, The more recently reported AREDS2 study showed some interesting parallels with this and other studies done over the past several years. While the fact that the significant improvements in macular protection effected with the addition of lutein, zeaxanthin, and fish oil to the original AREDS formulation did not make it into the abstract summary or into the primary analysis, a critical secondary analysis showed that there was a further reduction in the progression to advanced AMD when treated with these three additions/replacements to the original AREDS formulation.

In addition, this secondary analysis showed that when lutein and zeaxanthin replaced beta-carotene in the original AREDS supplement, there was further protection (18\%), and remarkably similar to this evaluation and others, there was an even greater protective value shown with the use of lutein and zeaxanthin in the lowest quartile of dietary intake of lutein and zeaxanthin recorded at baseline (26\%). This obviously makes quite a strong case for the use of these carotenoids with Omega 3, antioxidants, and zinc as a protective/preventive treatment against macular degeneration. While it is somewhat speculative, if the proper amounts of these two carotenoids were in the AREDS2 formula, and quite possibly if we began these treatments prior to detecting advanced degrees of dry macular changes, the AMD protective/preventive value would increase even further. More studies are needed, including earlier stages of the disease process and variations in the protective formulas studied, in order to present these supplements as a possible true preventive treatment regimen in a significant percentage of the population. This study, along with previously cited animal, epidemiological, and clinical human studies support this as a significant possibility if not a probability.

Until the past several years, we have not had the appropriate substantiating epidemiological or clinical studies that suggest diagnostic and treatment methods that might allow us to openly recommend these protocols that are now clearly called for in clinical practice. The importance of the initial data from the original AREDS and AREDS2 studies cannot be understated. The vastly expanded base of scientific knowledge, the strengthening of macular supplement formulas to include proper levels of carotenoids, better and safer antioxidants, the addition of Omega- 3 fatty acids, the development of MPOD as a critical clinical tool, and the improvements in OCT scanning technology offered by spectral domain OCT also cannot be understated. They can and will add to the armamentarium available to the clinician, greatly improving our capability to get an early start in our attempts to diminish the effects of the blinding disease of AMD within the patient population seen in primary eye care offices daily.

Final noteworthy comment: further evidence is supplied by a group not included in this evaluation, for obvious reasons. We have conducted and tracked MPOD and retinal evaluations in a group of 27 vegetarians during the study period, many more since, and their MPOD scores ranged between. 78 and .96, all showing no macular/ paramacular findings with SD-OCT. Proper diet is obviously a huge aid in prevention of AMD. There will perhaps be added medicinal treatments, one having already been shown to be viable in a very small sample size of cases, ${ }^{34}$ where statins were associated with reduction in retinal drusen, but the known side effects of very large quantities of systemic medications such as statins makes prevention with naturally-occurring formulations seem like the safest method of resolving the issue of $\mathrm{AMD}$, and possibly preventing it.

\section{Conclusion}

Increasing macular pigment density with ingestion of appropriate supplements containing zeaxanthin and lutein in the proper ratio (which may need to be varied in individual patient groups), together with Omega 3, antioxidants and zinc, can induce positive changes in the appearance of the central retina, with reduced drusen count, improved macular pigment density, and improvement in RPE viability. This increase in macular pigment density, as measured with flicker fusion based MPOD, improves visual acuity and visual functions. The results of this clinical case study demonstrate improvements in retinal changes from the early to moderate stages of dry AMD, and suggests that addressing this disease process in the earliest stages may have the greatest benefit.

Protection from the onset and/or advancement of macular changes that can lead to wet macular changes may be an added benefit that 
needs to be further investigated. Macular pigment optical densitometry (MPOD) testing is a very valuable, if not critical, clinical tool for not only detecting low levels of macular pigment density, and is essential in identifying the population at risk for this disease process, as well as giving the clinician the ability to follow the progress in treatment plans. When MPOD is coupled with SD-OCT evaluation of the macular region, the efficacy if the nutritional treatments installing or reversing disease progression can be accurately evaluated. The outcome statistics have demonstrated retinal protective capabilities with the use of the critical inclusion of carotenoid pigment/Omega-3 fatty acid/antioxidant/zinc formulas along with improved daily dietary intake of foods high in carotenoid value and sun protection.

\section{Funding}

None.

\section{Acknowledgments}

None.

\section{Conflicts of interest}

The authors declare that there was no conflict of interest.

\section{References}

1. Cabrera MP, Chihuailaf RH. Antioxidants and the integrity of ocular tissues. Vet Med Int. 2011.

2. Mares-Perlman JA, Klein R. Diet and age-related macular degeneration. In: Taylor A, Editors. Nutritional and Environmental Influences on the Eye. CRC Press: Boca Raton; 1999. p. 181-214.

3. Yuldirim Z, Ucgun NI, Yildirim F. The role of oxidative stress and antioxidants in the pathogenesis of age-related macular degeneration. Clinics (Sao Paulo). 2011;66(5):743-746.

4. Gorgels TG, van Norren D. Ultraviolet and green light cause different types of damage in rat retina. Invest Ophthalmol Vis Sci. 1995;36(5):851863.

5. Organisciak D, Darrow R, Barsalou L, et al. Light history and agerelated changes in retinal light damage. Invest Ophthalmol Vis Sci. 1998;39(7):1107-1116.

6. Sujak A, Gabrielska J, Grudzinski W, et al. Lutein and zeaxanthin as protectors of lipid membranes against oxidative damage: the structural aspects. Arch Biochem Biophys. 1999;371(2):301-307.

7. Age-Related Eye Disease Study Research Group. A randomized, placebo-con

8. trolled, clinical trial of high-dose supplementation with vitamins $\mathrm{C}$ and $\mathrm{E}$, beta carotene, and zinc for age-related macular degeneration and vision loss: AREDS report No. 8. Arch Ophthalmol. 2001;119(10):1417-1436.

9. Seddon JM, Ajani UA, Sperduto RD, et al. Dietary carotenoids, vitamins $\mathrm{A}, \mathrm{C}$, and $\mathrm{E}$, and advanced age-related macular degeneration. Eye Disease Case-Control Study Group. JAMA. 1994;272(18):1413-1420.

10. Mares JA, LaRowe TL, Snodderly DM, et al. Predictors of optical density of lutein and zeaxanthin in retinas of older women in the Carotenoids in Age-Related Eye Disease Study, an ancillary study of the Women's Health Initiative. Am J Clin Nutr. 2006;84(5):1107-1122.

11. Bone RA, Landrum JT, Mayne ST, et al. Macular pigment in donor eyes with and without AMD: a case control study. Invest Ophthalmol Vis Sci. 2001;42(1):235-240.
12. Mangels AR, Holden JM, Beecher GR, et al. Carotenoid content of fruits and vegetables; An evaluation of analytical data. $J$ Am Diet Assoc. 1993;93(3):284-296.

13. Perry A, Rasmussen H, Johnson EJ. Xanthophyll (lutein, zeaxanthin) content in fruits, vegetables, and corn and egg products. Journal of Food Composition and Analysis. 2009;22(1):9-15.

14. Bone RA, Landrum JT, Fernandez L, et al. Analysis of the macular pigment by HPLC: retinal distribution and age study. Invest Ophthal Vis Sci. 1998;29(6):842-849.

15. Johnson EJ, Maras JE, Rasmussen HM, et al. Intake of lutein and zeaxanthin differ with age, sex, and ethnicity. $J$ AM Diet Assoc. 2010;110(9):1357-1362.

16. SanGiovanni JP, Chew EY, Clemons TE, et al. for the Age-Related Eye Disease Study Research Group. The relationship of dietary carotenoid and vitamin $\mathrm{A}, \mathrm{E}$, and $\mathrm{C}$ intake with age-related macular degeneration in a case-control study: AREDS Report No. 22. Arch Ophthalmol. 2007;125(9):1225-1232.

17. Richer S, Stiles W, Statkute L, et al. Double-masked, placebo-controlled, randomized trial of lutein and antioxidant supplementation in the intervention of atrophic age-related macular degeneration: the Veterans LAST study (Lutein Antioxidant Supplementation Trial). Optometry. 2004;75(4):216-230.

18. Richer S, Devenport J, Lang JC. LAST II: Differential temporal response of macular pigment optical density in patients with atrophic age-related macular degeneration to dietary supplementation with xanthophylls. Optometry. 2007;78(5):213-219.

19. Age-Related Eye Disease Study (AREDS2) Research Group. Lutein + Zeaxanthin and Omega-3 Fatty Acids for Age-Related Macular Degeneration: the Age-Related Eye Disease Study 2 (AREDS2) randomized clinical trial. JAMA. 2013;309(19):2005-2015.

20. Delcourt C, Carrriere I, Delage M, et al. Plasma lutein and zeaxanthin and other carotenoids as modifiable risk factors for age-related maculopathy and cataract: the POLA study. Invest Ophthalmol Vis Sci. 2006;47:23292335 .

21. Richer S, Park DW, Epstein R, et al. Macular re-pigmentation enhances driving vision in elderly adult males with macular degeneration. $J$ Clin Exp Ophthalmol. 2012;3:3.

22. Stringham JM, Bovier ER, Wong JC, et al. The influence of dietary lutein and zeaxanthin on visual performance. $J$ Food Sci. 2009;75(1):R24-29.

23. Stringham JM, Fuld K, Wenzel AJ. Action spectrum for photophobia. $J$ Opt Soc Am A Opt Image Sci Vis. 2003;20(10):1852-1858.

24. Stringham JM, Fuld K. Wenzel AJ. Spatial properties of photophobia. Invest Ophthalmol Vis Sci. 2004;45(10):3838-3848.

25. Wenzel AJ, Fuld K, Stringham JM, et al. Macular pigments optical density and photophobia light threshold. Vis Res. 2006;46(28):4615-4622.

26. Stringham JM, Hammond BR. The glare hypothesis of macular pigment function. Opt Vis Sci. 2007;84(9):859-864.

27. Stringham JM, Hammond BR. Macular pigment and visual performance under glare conditions. Opt Vis Sci. 2008;85(2):82-88.

28. Stringham JM, Bovier ER, Wong JC, et al. The influence of dietary lutein and zeaxanthin in visual performance. J Food Sci. 2009;75(1):R24-29.

29. Stringham JM, Garcia PV, Smith PA, et al. Macular pigment and visual performance in glare: benefits for photostress recovery, disability glare, and visual discomfort. Invest Ophthal Vis Sci. 2011;52(10):7406-7415.

30. Snodderly DM, Stringham JM. Macular pigment reduces visual discomfort. ARVO Abstr Invest Ophthal Vis Sci. 2010;10:413. 
31. Ma L, Dou HL, Huang YM, et al. Improvement of retinal function in early age-related macular degeneration after lutein and zeaxanthin supplementation. Am Journal of Ophthalmol. 2012;155(10):625-633.

32. Bone RA, Landrum JT, Cao Y, et al. Macular pigment response to a supplement containing meso-zeaxanthin, lutein and zeaxanthin. Nutr Metab (Lond). 2007;4:12.

33. Wooten BR, Hammond BR, Land RI, et al. A practical method for measuring macular pigment optical density. Invest Ophthalmol Vis Sci. 1999;40:2481-2489.
34. deKlinkelder R, van der Veen RLP, Verbaak FD, et al. Macular pigment optical density measurements: evaluation of a device using heterochromic flicker photometry. Eye (Lond). 2011;25(1):367-375.

35. Vavvas DG, Daniels AB, Kapsala ZG, et al. Regression of some high-risk features of age-related macular degeneration (AMD) in patients receiving statin treatment. EBioMedicine. 2016;5:198-203. 